\title{
PA-055 LOW FALSE RECENT RATE OF LIMITING ANTIGEN AVIDITY ASSAY COMBINED WITH HIV-1 RNA DATA IN BOTSWANA
}

\author{
Sikhulile Moyo, ${ }^{1}$ Kenanao Kotokwe, ${ }^{1}$ Terence Mohammed, ${ }^{1}$ Coretah Boleo, ${ }^{1}$ \\ Lucy Mupfumi, ${ }^{1}$ Samuel Chishala, ${ }^{1}$ Simani Gaseitsiwe, ${ }^{1}$ Lesedi Tsalaile, ${ }^{1}$ \\ Herman Bussmann, ${ }^{1}$ Joseph Makhema, ${ }^{2}$ Marianna Baum, ${ }^{3}$ Richard Marlink, ${ }^{2}$ \\ Susan Engelbretch, ${ }^{4}$ Max Essex, ${ }^{2}$ Vladimir Novitsky ${ }^{2}$. ${ }^{1}$ BHP, Botswana; ${ }^{2}$ Harvard T.H. \\ Chan School of Public Health, United States of America; ${ }^{3}$ FIU-CHUA, United States \\ of America; ${ }^{4}$ Stellenbosch University, South Africa
}

\subsection{6/bmjgh-2016-000260.88}

Background Cross-sectional tests for recency of HIV infection are increasing in utility for estimating HIV incidence and evaluating impact of interventions. However, they have been shown to misclassify individuals with long standing infection as recent. Local performance characteristics are essential for their application. We estimated the false recency rate (FRR) among long term HIV-1 infected individuals from Botswana.

Methods A total of 1036 specimens from treatment naïve individuals known to be HIV-infected 1.5 to 2 years from baseline were tested using the limiting antigen-avidity assay (LAg) using a cut-off of 1.5 normalised optical density units (OD-n). Study participants were enrolled in HIV disease progression and did not qualify for treatment according to national guidelines at the time of enrolment. Baseline HIV status was determined using double ELISA. Viral and CD4 measures were done every 3 months.

Results Most participants were females (74.8\%) and median age was 35 years (IQR 30-42). The median CD4 cell count and viral load were 394 cells/ $\mathrm{LL}$ (IQR 303-524) and 4.25 copies/ $\mathrm{mL}$ (IQR 3.51-4.87), respectively. Overall the FRR was $0.97 \%$ (10/1036; 95\% CI: 0.46-1.77). Four samples had viral loads $>1000$ copies $/ \mathrm{mL}$, giving an adjusted FRR of $0.39 \%(4 / 1036$; 95\% CI 0.11-0.99).

Conclusions LAg had a very low FRR in this Botswana population using the algorithm involving viral load. We found viral load to be a complementary marker for improving the specificity of the LAg-avidity assay. To our knowledge, this is the first report of LAg-avidity FRR for the Botswana population, which is much lower than the $2 \%$ recommended by the WHO Incidence Assays Working Group. 